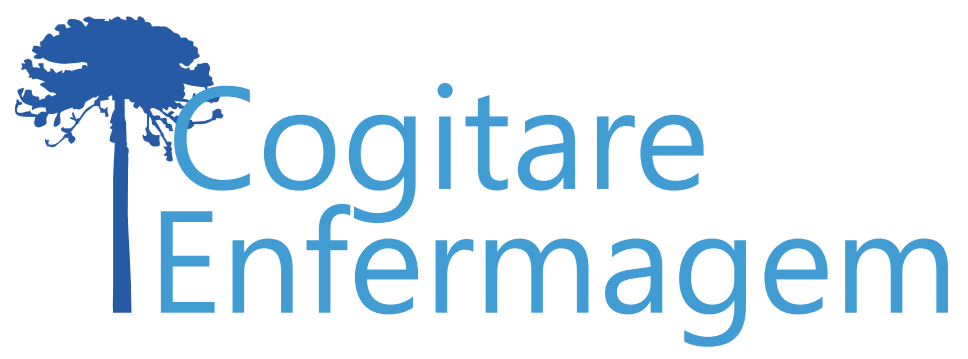

\title{
AMBIENTE DE PRÁTICA E A SEGURANÇA DO DOENTE NO BLOCO OPERATÓRIO: DIMENSÕES PREDITORAS
}

\author{
PRACTICE ENVIRONMENT AND PATIENT SAFETY IN THE \\ OPERATING ROOM: PREDICTIVE DIMENSIONS
}

\author{
Ana Sofia de Carvalho Mota ${ }^{1}$ (1) \\ Amélia Filomena de Oliveira Mendes Castilho ${ }^{2}$ (1) \\ Maria Manuela Ferreira Pereira Martins ${ }^{3}$ (i)
}

\begin{abstract}
Objectives: to identify the practice environment perceived by perioperative nurses and determine the dimensions of the practice environment that predict patient safety. Method: descriptive and explanatory study, in a sample of 1,001 nurses from 24 Portuguese hospitals (January to October 2018), using the Patient Safety in the Operating Room questionnaire and the Portuguese version of the Practice Environment Scale - Work Nursing Index. Use of descriptive analysis, Cronbach's alpha and stepwise method in the analysis of the linear regression model. Results: the global average $(M=2.49)$ of the practice environment indicates its classification as unfavorable. Regression models reveal that all dimensions of the environment are predictors of patient safety in the operating room, highlighting the dimension "Nursing fundamentals for quality of care". Conclusion: this study highlighted the influence of the practice environment on patient safety in the perioperative context and the need to introduce improvements.
\end{abstract}

DESCRIPTORS: Working Environment; Patient safety; Operating Rooms, Perioperative Nursing; Perioperative Care.

COMO REFERENCIAR ESTE ARTIGO:

Mota AS de C, Castilho AF de OM, Martins MMFP. Ambiente de prática e a segurança do doente no bloco operatório: dimensões preditoras. Cogit. Enferm. [Internet]. 2021 [acesso em "colocar data de acesso, dia, mês abreviado e ano"]; 26. Disponível em: http://dx.doi.org/10.5380/ce.v26i0.82289. 
A preocupação com os ambientes de prática de enfermagem não é recente, iniciouse com Florence Nightingale, que reconheceu que as condições e as circunstâncias onde se desenvolve a prática são determinantes para os resultados do doente. Outras teóricas de enfermagem dão destaque nos seus modelos conceptuais à interação constante entre os humanos e os seus ambientes, como Myra Estrin Levine, Barbara Artinian, Imogene King, Callista Roy e Jean Watson ${ }^{(1)}$. Por sua vez, o modelo conceptual específico do contexto perioperatório, adotado pela Association of periOperative Registered Nurses, o Perioperative Patient Focused Model, reconhece como um dos quatro domínios centrais para a prestação de cuidados, o domínio sobre o sistema de saúde, o qual remete para o ambiente de prática, assumindo a sua influência sobre os cuidados perioperatórios ${ }^{(2)}$. O ambiente constitui um conceito central de enfermagem, integrando um dos quatro conceitos metaparadigmáticos (pessoa, saúde, ambiente e enfermagem)(1).

No princípio dos anos 80 do século $\mathrm{XX}$, a marcada rotatividade e escassez de enfermeiros nos Estados Unidos da América determinaram a necessidade de compreender os fatores distintivos e caraterísticas organizacionais que permitiam que alguns hospitais, identificados como hospitais Magnet, conseguissem recrutar e reter enfermeiros ${ }^{(3)}$. Estes hospitais apresentavam características organizacionais específicas: a descentralização da tomada de decisão ao nível da enfermagem; uma forte e eficaz liderança de enfermagem; - reconhecimento da autonomia profissional de enfermagem, accountability e responsabilidade pela gualidade dos cuidados ao cliente; adequação dos recursos humanos e horários flexíveis ${ }^{(3)}$. É neste contexto que surge a noção que a crescente carência de enfermeiros não poderia ser revertida sem ambientes de trabalho que apoiem a prática de enfermagem. O ambiente de prática é definido como as características organizacionais que facilitam ou restringem a prática profissional de enfermagem ${ }^{(3)}$.

Em 2004, com a publicação do relatório "Keeping patients safe: Transforming the work environment of nurses" pelo Institute of Medicine (IOM), o ambiente de prática de enfermagem é assumido como um fator crítico na segurança do doente (SD), reconhecendose a necessidade da sua transformação para melhorar a $S^{(4)}$. Desde então, tem sido produzida evidência crescente indicando que o desenvolvimento de ambientes favoráveis à prática de enfermagem influencia os resultados clínicos dos doentes, nomeadamente no que se refere à redução da mortalidade, erros de medicação, infecções associadas aos cuidados de saúde, úlceras de pressão, e ocorrência de quedas ${ }^{(5-8)}$.

Um estudo que envolveu 535 hospitais americanos, 53.699 enfermeiros e 805.811 doentes, que analisou a evolução do ambiente de prática dos enfermeiros entre 2005 e $2016^{(9)}$, revelou que sete em cada 10 hospitais não registaram melhorias no ambiente da prática e que cerca de $10 \%$ registou uma deterioração dos ambientes, indiciando a existência de fragilidades e pouco investimento nos ambientes de prática. Os autores alertam para as consequências da falha na melhoria dos ambientes de prática, argumentando que ambientes de trabalho inadequados podem prejudicar o progresso na SD e dificultar a adesão às intervenções de segurança baseadas na evidência, potenciando a lacuna entre o conhecimento e a prática na prevenção de danos no doente ${ }^{(9)}$.

O bloco operatório (BO) constitui-se como um dos locais mais complexos de prestação de cuidados e onde ocorre o maior número de eventos adversos ${ }^{(10)}$. A implementação de projetos de segurança neste contexto, nomeadamente o projeto "Cirurgia Segura, Salva Vidas", tem-se confrontado com dificuldades de implementação a nível nacional e internacional, podendo comprometer a $\mathrm{SD}^{(10-11)}$. Assumindo que a otimização dos ambientes de prática de enfermagem tem potencial para contribuir positivamente para a SD e reconhecendo a lacuna na literatura no que se refere à caracterização dos ambientes de prática no contexto perioperatório, constituem-se como objetivos deste estudo identificar o ambiente de prática percebido pelos enfermeiros perioperatórios e determinar as dimensões do ambiente de prática preditoras da SD. 
Estudo descritivo e explicativo, inserido numa investigação inicial(12-13) utilizando a mesma amostra e procedimentos ético-legais. A população alvo é constituída por 2.975 enfermeiros perioperatórios a exercer funções em BOs (de doentes adultos) de hospitais do Serviço Nacional de Saúde português.

Foi realizada uma amostragem por clusters, tendo sido selecionados hospitais dos diferentes grupos de benchmarking da Administração Central dos Serviços de Saúde (ACSS) e Administrações Regionais de Saúde (ARS)(10,14). Integraram a amostra 24 hospitais, com uma percentagem por grupo de benchmarking, entre 50\% (grupos B e E) e 66,7\% (grupo F) e por ARS entre $42,86 \%$ (Lisboa e Vale do Tejo) e 100\% (Algarve), correspondendo a um total de 46 BOs.

Definiu-se como critério de inclusão ter tempo de atividade profissional superior a seis meses e como critérios de exclusão: exercer funções como enfermeiro(a) gestor(a) e estar ausente temporariamente do serviço no período da colheita de dados por atestado médico, licença de férias ou outra. A colheita de dados decorreu entre janeiro e outubro de 2018. Foram entregues 1.798 questionários, abrangendo todos os enfermeiros perioperatórios que cumpriam os critérios de inclusão. Responderam ao questionário 1.001 enfermeiros, correspondendo a uma taxa de resposta de $55,70 \%$.

A amostra, constituída por 1.001 participantes, possui um erro amostral estimado de $2,6 \%$, com intervalo de confiança de $95 \%$. A maioria dos participantes são do sexo feminino, correspondendo a 852 indivíduos $(84,90 \%)$. Os inquiridos apresentam uma média de idades de 42,74 anos ( $D P=0,27)$ e uma média de 19,76 anos ( $D P=0,27)$ de tempo de exercício. Estes enfermeiros possuem um tempo de experiência em $\mathrm{BO}$ e no atual serviço respectivamente de 13,52 anos $(\mathrm{DP}=0,28)$ e 11,56 anos $(\mathrm{DP}=0,27)$. Relativamente ao grau académico, 866 são licenciados $(79,10 \%), 122(18,50 \%)$ são mestres, oito $(1,90 \%)$ são bacharéis e cinco $(0,50 \%)$ são doutorados. Apenas 180 respondentes $(17,90 \%)$ possuem o título de especialista. 711 dos inquiridos trabalha em BOs centrais $(76,9 \%), 156(15,60 \%)$ em BOs de cirurgia de ambulatório e $74(7,40 \%)$ em BOs periféricos. $599(59,7 \%)$ participantes exercem a sua atividade em $\mathrm{BOs}$ acreditados/certificados.

Como instrumento de colheita de dados, utilizamos o questionário de Segurança do Doente no BO (SDBO) ${ }^{(12)}$ e a versão portuguesa do PES-NWII ${ }^{(15)}$. O questionário SDBO é constituído por 79 itens, pontuáveis numa escala tipo Likert de um (nunca) a cinco (sempre), que permitem avaliar 19 dimensões de SD, avaliando a implementação de boas práticas e auditorias no âmbito de nove áreas da SD.

O ambiente de prática foi avaliado através da versão portuguesa do PES-NW/ (15), constituída por 31 itens agrupados nas cinco subescalas da versão original do instrumento(3)'. Eliminamos o item 30 "A atribuição dos cuidados aos doentes promove a continuidade dos cuidados, isto é, o mesmo enfermeiro pode cuidar do mesmo doente de um dia para outro" da dimensão "Fundamentos de enfermagem para a qualidade dos cuidados", uma vez que este item não se aplica ao contexto de $\mathrm{BO}$, onde foi desenvolvida a presente investigação. O instrumento encontra-se sob a forma de escala de likert, graduada em quatro níveis, pontuados de um (concordo completamente) a quatro (discordo completamente). As respostas foram invertidas de forma a que os valores mais elevados da escala indiquem concordância. O ponto de corte da escala, para considerar ambiente de prática de enfermagem favorável, é $2,5^{(3)}$.

Os dados foram analisados com o programa informático IBM SPSS Statistics, versão 25.0. Na análise descritiva dos dados, calculamos as frequências (absolutas e percentuais), medidas de tendência central (média, máximo e mínimo) e medidas de dispersão (desvio padrão). Na avaliação da fiabilidade, calculamos a consistência interna de cada dimensão pelo coeficiente alf́a de Cronbach (a). Procedemos à análise do modelo de regressão linear múltipla, através do método stepwise. 
Foi solicitado parecer à Comissão de Ética da Unidade de Investigação em Ciências da Saúde da Enfermagem da Escola Superior de Enfermagem de Coimbra e realizados pedidos de autorização de recolha de informação aos conselhos de administração (CA) dos hospitais envolvidos no estudo. Obteve-se parecer positivo ( $P$ 458-09-2017) por parte da Comissão de Ética supracitada e dos CA dos 24 hospitais. Os questionários foram entregues em envelopes abertos, com explicitação dos objetivos e solicitação de consentimento informado. Foram posteriormente devolvidos em envelope fechado e o consentimento informado foi recolhido em separado.

\section{RESULTADOS}

As dimensões do ambiente de prática calculadas ${ }^{(3,15)}$ apresentaram valores de a que variam entre 0,74 ("Adequação dos recursos humanos e materiais") e 0,86 ("Participação nas políticas do hospital"), como podemos observar na Tabela 1, indiciando consistência interna adequada. A consistência interna global da escala revelou-se muito boa, com um valor de a de 0,93.

Tabela 1 - Análise descritiva e alfa de Cronbach das dimensões da versão portuguesa do PES-NWI: itens, mínimo, máximo, média e desvio padrão $(n=1.001)$. Coimbra, Portugal, 2021

\begin{tabular}{|c|c|c|c|c|c|c|c|}
\hline Dimensões & Itens & $\mathbf{n}$ & $\alpha$ & Mín & Máx & $\mathbf{M}$ & DP \\
\hline $\begin{array}{l}\text { Participação nas políticas do hospital } \\
\text { (PPH) }\end{array}$ & $\begin{array}{r}5 ; 6 ; 17 ; 11,15 ; \\
21 ; 23 ; 27 ; 28 \\
\end{array}$ & 987 & 0,86 & 1 & 4 & 2,21 & 0,54 \\
\hline $\begin{array}{l}\text { Fundamentos de enfermagem para a } \\
\text { qualidade dos cuidados (FEQC) }\end{array}$ & $\begin{array}{r}4 ; 14 ; 18 ; 19,22 ; \\
25 ; 26 ; 29 ; 31 \\
\end{array}$ & 983 & 0,81 & 1 & 4 & 2,66 & 0,49 \\
\hline $\begin{array}{l}\text { Capacidade de gestão, liderança e de } \\
\text { apoio dos enfermeiros (CGLAE) }\end{array}$ & $3 ; 7 ; 10 ; 13 ; 20$ & 986 & 0,82 & 1 & 4 & 2,43 & 0,62 \\
\hline $\begin{array}{l}\text { Adequação dos recursos humanos e } \\
\text { materiais (ARHM) }\end{array}$ & $1 ; 8 ; 9 ; 12$ & 987 & 0,74 & 1 & 4 & 2,38 & 0,65 \\
\hline $\begin{array}{l}\text { Relação colegial entre médicos e } \\
\text { enfermeiros (RCME) }\end{array}$ & $2 ; 16 ; 24$ & 996 & 0,77 & 1 & 4 & 2,79 & 0,51 \\
\hline
\end{tabular}

PES-NWI global $\quad 0,93 \quad 2,49$

$\mathrm{n}=$ Amostra; $\mathrm{a}=$ alfa de Cronbach Mín = Mínimo; Máx = Máximo; $M$ = Média; DP = Desvio padrão.

Fonte: Autores (2021).

Constatou-se uma heterogeneidade de respostas obtidas, com respostas em todos os pontos da escala em todos os itens. Apenas duas dimensões apresentaram valores superiores ao ponto médio da escala $(M \geq 2,5)$ : "Relação colegial entre médicos e enfermeiros" ( $M=2,79 ; \mathrm{DP}=0,51)$ e "Fundamentos de enfermagem para qualidade dos cuidados" (M=2,66; DP=0,49). A dimensão "Participação nas políticas do hospital" reuniu apreciação menos favorável dos enfermeiros $(M=2.21 ; \mathrm{DP}=0,54)$, seguido da "Adequação dos recursos humanos e materiais" $(M=2,38 ; \mathrm{DP}=0,65)$ e da "Capacidade de gestão, liderança e de apoio dos enfermeiros" ( $M=2,43 ; \mathrm{DP}=0,62)$. Foi igualmente calculada a média global de concordância dos enfermeiros com o ambiente de prática $(M=2,49)$, que não atingiu o ponto médio da escala, corroborando a existência de fragilidades no ambiente de prática. 
Com o intuito de analisar o poder explicativo do ambiente de prática na SD no BO, recorremosàtécnica de regressãolinear múltipla. Consideramos comovariáveis dependentes as 19 dimensões do questionário SDBO e como variáveis preditoras as cinco dimensões do ambiente de prática ${ }^{(3,15)}$. Na Tabela 2 podemos observar que todas as dimensões do ambiente de prática são preditoras da SD no BO. Os modelos têm maior poder explicativo nas dimensões "Prevenção e controle de infeção e resistências aos antimicrobianos formação e vigilância epidemiológica" (30\%), "Cultura de segurança do ambiente interno" (26\%), "Segurança cirúrgica - auditorias" (26\%), "Segurança da comunicação - auditorias" (26\%) e "Análise e prevenção de incidentes" (25\%). Contrariamente, revelam menor poder explicativo nas dimensões "Segurança na utilização da medicação - prescrição" (7\%), "Prevenção de úlceras de pressão - boas práticas" (10\%), "Identificação inequívoca - boas práticas" (11\%), "Notificação de incidentes" (12\%), "Prevenção de úlceras de pressão auditorias" (12\%), e "Prevenção de úlceras de pressão - recursos" (13\%).

Tabela 2 - Dimensões do Ambiente de Prática que entram nos modelos preditores das dimensões de SD no BO: pesos da regressão Beta ajustado, testes $t$, e percentagem explicativa de cada modelo $(n=1.001)$.

Coimbra, Portugal, 2021 (continua)

\begin{tabular}{|c|c|c|c|c|c|}
\hline $\begin{array}{l}\text { Dimensões da Segurança do Doente } \\
\text { no Bloco Operatório }\end{array}$ & $\begin{array}{l}\text { Ambiente } \\
\text { de prática } \\
\text { (Dimensões } \\
\text { Preditoras) }\end{array}$ & $\begin{array}{c}\text { Pesos da } \\
\text { regressão } \\
\text { Beta } \\
\text { ajustado }\end{array}$ & $\mathbf{T}$ & $\mathbf{P}$ & $\begin{array}{c}\% \\
\text { explicada }\end{array}$ \\
\hline \multirow[t]{2}{*}{ Cultura de segurança } & FEQC & 0,35 & $-9,57$ & 0 & \multirow[t]{2}{*}{26} \\
\hline & CGLAE & 0,21 & $-5,58$ & 0 & \\
\hline $\begin{array}{l}\text { Segurança da comunicação - boas } \\
\text { práticas }\end{array}$ & FEQC & 0,44 & 14,99 & 0 & 19 \\
\hline \multirow{4}{*}{$\begin{array}{l}\text { Segurança da comunicação - } \\
\text { auditorias }\end{array}$} & $\mathrm{PPH}$ & 0,2 & 4,01 & 0 & \multirow[t]{4}{*}{26} \\
\hline & FEQC & 0,24 & 5,65 & 0 & \\
\hline & CGLAE & 0,19 & 4,16 & 0 & \\
\hline & ARHM & $-0,09$ & $-2,66$ & 0,01 & \\
\hline \multirow[t]{2}{*}{ Segurança cirúrgica - boas práticas } & CGLAE & 0,25 & 6,17 & 0 & \multirow[t]{2}{*}{16} \\
\hline & FEQC & 0,19 & 4,84 & 0 & \\
\hline \multirow[t]{3}{*}{ Segurança cirúrgica - auditorias } & FEQC & 0,28 & 6,54 & 0 & \multirow[t]{3}{*}{26} \\
\hline & CGLAE & 0,18 & 4,11 & 0 & \\
\hline & $\mathrm{PPH}$ & 0,11 & 2,19 & 0,03 & \\
\hline \multirow{3}{*}{$\begin{array}{l}\text { Segurança na utilização de medicação } \\
\text { - boas práticas }\end{array}$} & FEQC & 0,24 & 5,42 & 0 & \multirow[t]{3}{*}{16} \\
\hline & CGLAE & 0,01 & 2,13 & 0,03 & \\
\hline & $\mathrm{PPH}$ & 0,11 & 2,12 & 0,03 & \\
\hline \multirow{4}{*}{$\begin{array}{l}\text { Segurança na utilização de medicação } \\
\text { - auditorias }\end{array}$} & $\mathrm{PPH}$ & 0,2 & 3,88 & 0 & \multirow[t]{4}{*}{21} \\
\hline & FEQC & 0,18 & 4,07 & 0 & \\
\hline & CGLAE & 0,18 & 3,97 & 0 & \\
\hline & ARHM (-) & $-0,09$ & $-2,37$ & 0,02 & \\
\hline \multirow{2}{*}{$\begin{array}{l}\text { Segurança na utilização da medicação } \\
\text { - prescrição }\end{array}$} & $\mathrm{PPH}$ & 0,16 & 3,39 & 0 & \multirow[t]{2}{*}{7} \\
\hline & FEQC & 0,14 & 3,08 & 0 & \\
\hline
\end{tabular}




\begin{tabular}{|c|c|c|c|c|c|}
\hline \multirow{2}{*}{$\begin{array}{l}\text { Identificação inequívoca - boas } \\
\text { práticas }\end{array}$} & FEQC & 0,3 & 8,56 & 0 & \multirow[t]{2}{*}{11} \\
\hline & RCME & 0,07 & 2,1 & 0 & \\
\hline \multirow{2}{*}{ Identificação inequívoca - auditorias } & $\mathrm{PPH}$ & 0,27 & 6,02 & 0 & \multirow[t]{2}{*}{14} \\
\hline & FEQC & 0,13 & 2,98 & 0 & \\
\hline \multirow[t]{3}{*}{ Prevenção de quedas - boas práticas } & FEQC & 0,34 & 7,83 & 0 & \multirow[t]{3}{*}{20} \\
\hline & CGLAE & 0,25 & 5,42 & 0 & \\
\hline & PPH (-) & $-0,12$ & $-2,36$ & 0 & \\
\hline \multirow[t]{3}{*}{ Prevenção de quedas - auditorias } & $\mathrm{PPH}$ & 0,17 & 3,29 & 0 & \multirow[t]{3}{*}{16} \\
\hline & FEQC & 0,17 & 3,73 & 0 & \\
\hline & CGLAE & 0,11 & 2,33 & 0,02 & \\
\hline \multirow{2}{*}{$\begin{array}{l}\text { Prevenção de úlceras de pressão - } \\
\text { boas práticas }\end{array}$} & FEQC & 0,22 & 5,32 & 0 & \multirow[t]{2}{*}{10} \\
\hline & CGLAE & 0,13 & 3,13 & 0 & \\
\hline \multirow{4}{*}{$\begin{array}{l}\text { Prevenção de úlceras de pressão - } \\
\text { auditorias }\end{array}$} & $\mathrm{PPH}$ & 0,25 & 4,62 & 0 & \multirow[t]{4}{*}{12} \\
\hline & ARHM & $-0,13$ & $-3,24$ & 0 & \\
\hline & FEQC & 0,1 & 2,24 & 0,03 & \\
\hline & CGLAE & 0,11 & 2,18 & 0,03 & \\
\hline \multirow{3}{*}{$\begin{array}{l}\text { Prevenção de úlceras de pressão - } \\
\text { recursos }\end{array}$} & CGLAE & 0,14 & 3,37 & 0 & \multirow[t]{3}{*}{13} \\
\hline & ARHM & 0,16 & 4,26 & 0 & \\
\hline & FEQC & 0,13 & 3,12 & 0 & \\
\hline \multirow[t]{2}{*}{ Notificação de incidentes } & FEQC & 0,21 & 4,61 & 0 & \multirow[t]{2}{*}{12} \\
\hline & $\mathrm{PPH}$ & 0,17 & 3,79 & 0 & \\
\hline \multirow[t]{2}{*}{ Análise e prevenção de incidentes } & FEQC & 0,36 & 9,62 & 0 & \multirow[t]{2}{*}{25} \\
\hline & CGLAE & 0,18 & 4,85 & 0 & \\
\hline \multirow{3}{*}{$\begin{array}{l}\text { Prevenção e controlo de infeção e } \\
\text { resistências aos antimicrobianos - boas } \\
\text { práticas }\end{array}$} & FEQC & 0,32 & 7,78 & 0 & \multirow[t]{3}{*}{19} \\
\hline & CGLAE & 0.11 & 2,75 & 0,01 & \\
\hline & RCME & 0.07 & 2,07 & 0,04 & \\
\hline \multirow{3}{*}{$\begin{array}{l}\text { Prevenção e controle de infeção e } \\
\text { resistências aos antimicrobianos - } \\
\text { formação e vigilância epidemiológica }\end{array}$} & FEQC & 0,37 & 9 & 0 & \multirow[t]{3}{*}{30} \\
\hline & CGLAE & 0,14 & 3,3 & 0 & \\
\hline & $\mathrm{PPH}$ & 0,1 & 2,14 & 0,03 & \\
\hline
\end{tabular}

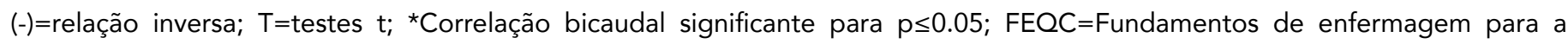
qualidade dos cuidados; CGLAE=Capacidade de gestão, liderança e de apoio dos enfermeiros; PPH=Participação nas políticas do hospital; $A R H M=$ Adequação dos recursos humanos e materiais; RCME=Relação colegial entre médicos e enfermeiros Fonte: Autores (2021).

Destaca-se o poder preditor da dimensão "Fundamentos de enfermagem para a qualidade dos cuidados", que entra em todos os modelos preditores com elevados valores de Beta, e das dimensões "Capacidade de gestão, liderança e de apoio dos enfermeiros" e "Participação nas políticas do hospital", que também entram na maioria dos modelos. Por sua vez, as dimensões "Relação colegial entre médicos e enfermeiros" e a "Adequação dos recursos humanos e materiais" revelam poder preditor mais baixo (Tabela 2). 
A escala utilizada na avaliação do ambiente de prática (PES-NWI) revelou-se um instrumento fiável em contexto perioperatório, tendo-se obtido valores de a, quer no global quer nas diferentes dimensões, superiores aos encontrados pelos autores da validação da escala para a população portuguesa ${ }^{(15)}$ e pela autora da versão original $\left.\right|^{(3)}$.

De acordo com a classificação proposta por Lake $e^{(3)}$, o ambiente de prática percebido pelos enfermeiros perioperatórios portugueses é considerado desfavorável, com valor médio global inferior a 2,5, alertando para a necessidade de melhorar a gestão do ambiente neste contexto. Estes resultados vão ao encontro de um estudo que envolveu uma amostra de 2.235 enfermeiros de serviços médico-cirúrgicos portugueses $(M=2,44)^{(16)}$, e dos resultados obtidos pelos autores da validação da escala para o contexto português $(n=365 ; M=2,57)^{(17)}$, que evidenciam fragilidades do ambiente de prática de enfermagem em diferentes contextos clínicos. A nível internacional, uma revisão da literaturaa ${ }^{(18)}$ revela que tem existido pouco investimento nos ambientes de prática, considerando que nos estudos analisados observaram-se valores médios globais do PES-NWI entre 2,30 e 3,07. Os resultados de um estudo que envolveu 535 hospitais Americanos, no período de 2005 a $2016^{(9)}$, evidenciaram que não se observaram melhorias no ambiente de prática na maioria dos hospitais estudados, e cerca de $10 \%$ apresentaram uma deterioração.

Os resultados desta investigação revelam que apenas duas dimensões foram classificadas positivamente: "Relação colegial entre médicos e enfermeiros" e "Fundamentos de enfermagem para qualidade dos cuidados", indo ao encontro de outras investigações ${ }^{(16,19)}$.

No que se refere à dimensão "Relação colegial entre médicos e enfermeiros" $(M=2,79)$, os valores moderadamente positivos percepcionados pelos enfermeiros perioperatórios portugueses permitem identificar boas relações, colaboração e trabalho em equipe entre enfermeiros e médicos, e são concordantes com os resultados obtidos noutros estudos analisados ${ }^{(16-17,19)}$. Os melhores resultados observados no contexto perioperatório, superiores aos identificados noutros contextos de cuidados, poderão estar associados ao fato de o trabalho em BO exigir grande esforço cooperativo da equipe e interação constante entre os diferentes grupos profissionais, que reconhecem a importância de boas relações e do trabalho interdisciplinar em contextos complexos, como é o caso do BO. Também os estudos nacionais que avaliam a cultura de segurança identificam o "Trabalho em equipe" como a dimensão com melhores resultados, quer nos diferentes contextos de cuidados ${ }^{(20)}$, quer ao nível dos serviços de $\mathrm{BO}^{(21)}$, corroborando o investimento dos profissionais neste domínio.

A dimensão "Fundamentos de enfermagem para qualidade dos cuidados" $(M=2,66)$ evidencia que os enfermeiros perioperatórios portugueses têm uma perspectiva positiva, ainda que moderada, do contributo da enfermagem para a melhoria da qualidade na sua organização. Os resultados alertam para a necessidade de maior acompanhamento e supervisão dos profissionais recém-admitidos nas organizações, bem como de maior foco na implementação consistente e sistemática de planos de cuidados, que promovam a qualidade e a continuidade dos cuidados.

Destacamos os resultados negativos observados na dimensão "Participação nas políticas do hospital" $(M=2,21)$, à semelhança dos resultados observados noutros estudos $^{(16-17,19)}$. Os resultados apontam para o desenvolvimento de estratégias de motivação intrínseca e extrínseca que promovam maior envolvimento dos enfermeiros nos processos de decisão, garantindo a integração da sua perspectiva e consequentemente uma maior adesão no cumprimento da política interna.

O valor médio observado na dimensão "Adequação dos recursos humanos e materiais" (M=2,38), embora ligeiramente superior aos observados noutros estudos ${ }^{(16-17,19)}$, indicia a precariedade na adequação de recursos humanos e materiais no $\mathrm{BO}$, ainda que 
menos acentuada do que noutros contextos hospitalares. A nível internacional, como revela a revisão da literatura de Swiger ${ }^{(18)}$, os baixos valores observados sistematicamente nesta dimensão alertam para uma área crítica do ambiente da prática. Também os resultados nacionais da avaliação da cultura de segurança revelam que a dimensão "Dotação de Recursos Humanos" se encontra entre as mais críticas, quer a nível hospitalar (20), quer em contexto específico de $\mathrm{BO}^{(21)}$, corroborando a necessidade de as organizações garantirem dotações seguras nos diferentes contextos de cuidados, inclusive no contexto perioperatório.

Os enfermeiros perioperatórios também percepcionam fragilidades na "Capacidade de gestão, liderança e de apoio dos enfermeiros" $(M=2,43)$, evidenciando um amplo espaço de melhoria, tal como identificado por outras investigações ${ }^{(16,19)}$, o que deve ser tido em conta pela gestão intermédia e gestão de topo das organizações hospitalares. Salienta-se nesta dimensão oportunidades de melhoria importantes na supervisão de cuidados.

Identificamos os modelos preditores da segurança dos doentes no BO e verificamos que todas as dimensões do ambiente de prática são preditoras da implementação de práticas de SD no BO, com destaque para as dimensões "Fundamentos de enfermagem para a qualidade dos cuidados", "Capacidade de gestão, liderança e de apoio dos enfermeiros" e "Participação nas políticas do hospital". Estes resultados reforçam a importância de um modelo e filosofia de enfermagem, da liderança de enfermagem, e do envolvimento dos enfermeiros nas decisões de políticas internas, como forma de promover a SD no contexto perioperatório. Os nossos resultados são corroborados por estudos em que a otimização dos ambientes de prática de enfermagem está associada a uma percepção mais positiva dos indicadores de segurança por parte dos profissionais ${ }^{(6,9)}$ e apresenta uma relação inversa significativa com o número de eventos adversos, erros de medicação, úlceras de pressão e quedas ${ }^{(5,7)}$.

Consideramos como limitação do presente estudo não termos incluído, para além da percepção dos profissionais sobre a implementação de boas práticas de SD, a avaliação de indicadores de resultados de SD.

\section{CONCLUSÃO}

O ambiente de prática, na perspectiva dos enfermeiros perioperatórios portugueses, apresenta fragilidades, alertando para a necessidade de um investimento importante nas diferentes dimensões. Os enfermeiros consideram de forma positiva, apesar da pouca expressão, as relações de trabalho com o grupo profissional médico e o seu envolvimento na melhoria da qualidade na sua organização, bem como identificam poucas oportunidades de desenvolvimento profissional e de participação em questões hospitalares, salientam a precariedade na adequação de recursos humanos e materiais e alertam para oportunidades de melhoria na gestão e liderança dos enfermeiros.

Os modelos de regressão revelam a influência positiva das dimensões do ambiente de prática na SD no BO, com destaque para as dimensões "Fundamentos de enfermagem para a qualidade dos cuidados" e "Capacidade de gestão, liderança e de apoio dos enfermeiros", sendo fundamental introduzir melhorias neste âmbito para melhorar a SD em contexto perioperatório.

Os resultados obtidos nesta investigação permitem, de forma pioneira, descrever o ambiente de prática de enfermagem no contexto perioperatório português, evidenciar a influência do ambiente de prática na SD neste contexto, e alertar para a necessidade de melhorar várias dimensões do ambiente da prática quando se pretende melhorar a implementação de estratégias promotoras da SD, contribuindo assim para o melhor conhecimento desta realidade. Estes resultados fornecem informações relevantes para a prática de enfermagem, para a gestão intermédia e gestão de topo das organizações 
hospitalares, desafiando os líderes de enfermagem a conduzirem uma transformação positiva dos ambientes de prática de enfermagem, com base na avaliação diagnóstica realizada.

Demonstrado o impacto do ambiente de prática nas diferentes dimensões de SD em contexto perioperatório, espera-se que este desenho de estudo possa ser replicado noutros contextos profissionais de enfermagem. Considera-se igualmente importante investigar o impacto de projetos de melhoria do ambiente de prática nos indicadores de resultado de SD.

\section{REFERÊNCIAS}

1. McEwen M, Wills EM. Bases teóricas de Enfermagem. 4. ed. (Tradução Garcez RM.). São Paulo, Brazil: ARTMED, 2016 (Obra original publicada em 2014). 608 p.

2. Rothorck JC, Smith DA. Selecting the perioperative patient focused model. AORN journal [Internet]. 2000 [acesso em 12 out. 2020]; 71(5) Disponível em: http://doi.org/10.1016/s0001-2092(06)61552-4.

3. Lake ET. Development of the practice environment scale of the nursing work index. Res Nurs Health [Internet]. 2002 [acesso em 12 out. 2020]; 25. Disponível em: http://doi.org/10.1002/nur.10032.

4. Institute of Medicine. Keeping patients safe: transforming the work environment of nurses [Internet]. Washington (DC), National Academies Press (US): page A.; 2004 [acesso em 12 out. 2020]. 462 p.

Disponível em: http://doi.org/10.17226/10851.

5. Cho $E$, Chin DL, Kim S, Hong $O$. The relationships of nurse staffing level and work environment with patient adverse events. J Nurs Scholarsh [Internet]. 2016. [acesso em 12 out. 2020]; 48(1). Disponível em: http://doi.org/10.1111/jnu.12183.

6. Lake ET, Hallowell SG, Kutney-Lee A, Hatfield LA, Guidice MD, Boxer BA, et al. Higher quality of care and patient safety associated with better NICU work environments. J Nurs Care Qual [Internet]. 2016 [acesso em 12 out. 2020]; 31(1). Disponível em: http://doi.org/10.1097/NCQ.0000000000000146.

7. Ma C, Park SH. Hospital magnet status, unit work environment, and pressure ulcers. J Nurs Scholarsh [Internet]. 2015[acesso em 12 out. 2020]; 47(6). Disponível em: http://doi.org/10.1111/jnu.12173.

8. Olds DM, Aiken LH, Cimiotti JP, Lake ET. Association of nurse work environment and safety climate on patient mortality: a cross-sectional study. Int J Nurs stud[Internet]. 2017 [acesso em 12 out. 2020]; 74 Disponível em: http://doi.org/10.1016/j.ijnurstu.2017.06.004.

9. Aiken LH, Sloane DM, Barnes H, Cimiotti JP, Jarrín OF, McHugh MD. Nurses' and patients' appraisals show patient safety in hospitals remains a concern. Health Affairs [Internet]. 2018 [acesso em 12 out. 2020] 37(11) Disponível em: http://doi.org/10.1377/h/thaff.2018.0711.

10. Ministério da Saúde. Despacho n ${ }^{\circ} 1400-A / 2015$ de 10 de fevereiro de 2015. [Internet]. Diário da República [acesso em 12 out. 2020]; $2^{a}$ Série, $n^{\circ}$ 28, 3882-(2)-3882-(10). Disponível em: https://dre.pt/ application/conteudo/66463212.

11. Organização Mundial de Saúde. Orientações da OMS para Cirurgia Segura 2009: cirurgia segura salva vidas, versão portuguesa [Internet]. Lisboa, Portugal: Direção Geral da Saúde, 2015 [acesso em 12 out. 2020]. 196 p. Disponível em: https://www.dgs.pt/ficheiros-de-upload-2/orientacoes-da-oms-para-acirurgia-segura-2009-pdf.aspx.

12. Mota AS de C, Castilho AF de OM. Construção e validação psicométrica do Questionário de Segurança do Doente no Bloco Operatório. Referência [Internet]. 2019 [acesso em 12 out. 2020]; 21 (2). Disponível em: https://doi.org/10.12707/RIV19012. 
13. Mota AS de C, Castilho AF de OM, Martins MMFP da S. Avaliação da segurança do doente no bloco operatório: perceção dos enfermeiros. Referência [Internet]. 2021 [acesso em 19 jun 2021]; 5(6). Disponível em: http://doi.org/10.12707/RV20134.

14. Ministério da Saúde. Avaliação da situação nacional dos blocos operatórios: relatório final. [Internet]. Lisboa, Portugal: Autor. 2015 [acesso em 12 out. 2020]. p. 273. Disponível em: http://www.apca.com.pt/ documentos/2015/Avaliacao situacao nacional_blocos_operatorios Outubro2015.pdf.

15. Amaral AFS, Ferreira PL, Lake ET. Validation of the practice environment scale of the nursing work index (PES-NWI) for the portuguese nurse population. Int J Caring Sci [Internet]. 2012 [acesso em 12 out. 2020]; 5(3). Disponível em: https://www.researchgate.net/ publication/267959485 O R I G I N A L P A P E R Validation of the Practice Environment Scale of the Nursing Work Index PES-NWI for the Portuguese nurse population.

16. Jesus EH de, Roque SMB, Amaral AFS. Estudo RN4CAST em Portugal: ambientes de prática de enfermagem. RIE [Internet]. 2015 [acesso em 12 out. 2020]; Disponível em: https://www.researchgate.net/ profile/Antonio-Amaral-4/publication/289537196_Estudo RN4Cast em_Portugal ambientes de pratica de enfermagem/links/568fd7bb08aed0aed810bbe0/Estudo-RN4Cast-em-Portugal-ambientes-de-praticade-enfermagem.pdf.

17. Amaral AFS, Ferreira PL, Vidinha TS, Cardoso MLS. A perceção dos enfermeiros acerca do ambiente de prática dos cuidados em quatro hospitais da região centro. RIE [Internet]. 2013 ago [acesso em 12 out. 2020]; 4(2). Disponível em: https://www.researchgate.net/publication/289670981 Percecao dos Enfermeiros Acerca do Ambiente da Pratica dos Cuidados em Quatro Hospitais da Regiao Centro.

18. Swiger PA, Patrician PA, Miltner RSS, Raju D, Breckenridge-Sproat S, Loan LA. The Practice Environment Scale of the Nursing Work Index: an updated review and recommendations for use. Int J nursing studi [Internet]. 2017 [acesso em 12 out. 2020]; 201774. Disponível em: http://dx.doi. org/10.1016/j.ijnurstu.2017.06.003.

19. Lake ET, Friese CR. Variations in nursing practice environments: Relations to staffing and hospital characteristics. Nurs Res [Internet]. 2006 [acesso em 12 out. 2020]; 55(1). Disponível em: http://doi. org/10.1097/00006199-200601000-00001.

20. Direção-Geral da Saúde (DGS). Relatório de Avaliação de Segurança dos Doentes: Avaliação da Cultura de Segurança dos Doentes[Internet]. Lisboa, Portugal: Autor, 2015 [acesso em 12 out. 2020]. 60 p. Disponível em: https://www.dgs.pt/documentos-e-publicacoes/relatorio-seguranca-dos-doentesavaliacao-da-cultura-nos-hospitais-pdf.aspx.

21. Mota ASC. Cultura de Segurança do Doente e Lista de Verificação de Segurança Cirúrgica: perceção dos profissionais do bloco operatório (Dissertação de mestrado). [Coimbra]: Escola Superior de Enfermagem de Coimbra, 2015. 238p 


\title{
AMBIENTE DE PRÁTICA E A SEGURANÇA DO DOENTE NO BLOCO OPERATÓRIO: DIMENSÕES PREDITORAS
}

\section{RESUMO:}

Objetivos: identificar o ambiente de prática percepcionado pelos enfermeiros perioperatórios e determinar as dimensões do ambiente de prática preditoras da segurança do doente. Método: estudo descritivo e explicativo, numa amostra de 1.001 enfermeiros de 24 hospitais portugueses (janeiro a outubro de 2018), utilizando o questionário Segurança do Doente no Bloco Operatório e a versão portuguesa do Practice Environment Scale - Work Nursing Index. Recurso à análise descritiva, alfa de Cronbach e método stepwise na análise do modelo de regressão linear. Resultados: a média global $(M=2,49)$ do ambiente de prática indicia sua classificação como desfavorável. Os modelos de regressão revelam que todas as dimensões do ambiente são preditoras da segurança do doente no bloco operatório, destacando-se a dimensão "Fundamentos de enfermagem para qualidade dos cuidados". Conclusão: este estudo permitiu evidenciar a influência do ambiente de prática na segurança do doente no contexto perioperatório e a necessidade de introdução de melhorias.

DESCRITORES: Ambiente de Trabalho; Segurança do Paciente; Salas Cirúrgicas, Enfermagem Perioperatória; Cuidados Perioperatórios.

\section{AMBIENTE DE TRABAJO Y LA SEGURIDAD DEL PACIENTE EN EL QUIRÓFANO: DIMENSIONES PREDICTIVAS}

\section{RESUMEN:}

Objetivos: identificar el ambiente de trabajo percibido por enfermeras perioperatorias y determinar las dimensiones del ambiente de trabajo que predicen la seguridad del paciente. Método: estudio descriptivo y explicativo, en una muestra de 1.001 enfermeros de 24 hospitales portugueses (enero a octubre de 2018), utilizando el cuestionario Seguridad del Paciente en el Quirófano y la versión portuguesa de la Escala de Ambiente de Trabajo - Índice de Enfermería Laboral. Utilización de análisis descriptivo, alfa de Cronbach y método paso a paso en el análisis del modelo de regresión lineal. Resultados: la media global $(M=2,49)$ del ambiente de trabajo indica su clasificación como desfavorable. Los modelos de regresión revelan que todas las dimensiones del ambiente de trabajo son predictores de la seguridad del paciente en el quirófano, destacando la dimensión "Fundamentos de enfermería para la calidad asistencial". Conclusión: este estudio mostró la influencia del ambiente de trabajo en la seguridad del paciente en el contexto perioperatorio y la necesidad de introducir mejoras.

DESCRIPTORES: Ambiente de Trabajo; Seguridad del Paciente; Quirófanos, Enfermería Perioperatoria; Atención Perioperativa.

Recebido em: 02/08/2021

Aprovado em: 03/10/2021

Editora associada: Luciana Puchalski Kalinke

\author{
Autor Correspondente: \\ Ana Sofia de Carvalho Mota \\ Universidade do Porto - Porto, Portugal \\ E-mail: anasofiacarvalhomota@esenfc.pt
}

Contribuição dos autores:

Contribuições substanciais para a concepção ou desenho do estudo; ou a aquisição, análise ou interpretação de dados do estudo - Mota AS de C, Castilho AF de OM; Elaboração e revisão crítica do conteúdo intelectual do estudo - Mota AS de C, Castilho AF de OM. Todos os autores aprovaram a versão final do texto. permite o uso irrestrito, a distribuição e reprodução em qualquer meio desde que o artigo original seja devidamente citado. 